This article was downloaded by: [V. Yu. Topolov]

On: 17 September 2012, At: 06:22

Publisher: Taylor \& Francis

Informa Ltd Registered in England and Wales Registered Number: 1072954 Registered office: Mortimer House, 37-41 Mortimer Street, London W1T 3J H, UK

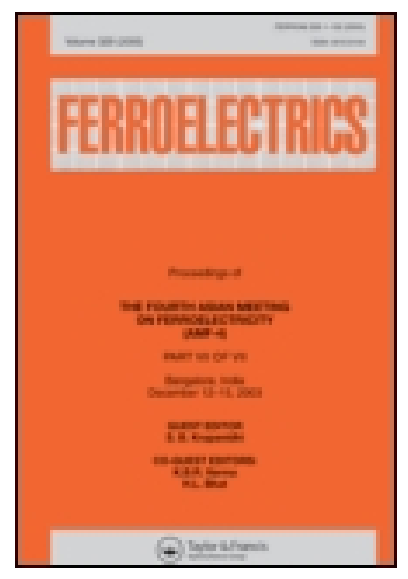

\title{
Ferroelectrics
}

Publication details, including instructions for authors and subscription information:

http:// www. tandfonline.com/loi/ gfer20

\section{Anisotropy Factors and Hydrostatic Parameters of 1-3-Type Piezo-Active Composites with Auxetic Polymer Matrices}

\author{
V. Yu. Topolov ${ }^{\text {a }}$, S. E. Filippov ${ }^{b} \&$ P. Bisegna ${ }^{c}$ \\ ${ }^{a}$ Department of Physics, Southern Federal University, 344090 \\ Rostov-on-Don, Russia \\ b Scientific Design \&Technology Institute "Piezopribor", Southern \\ Federal University, 344090 Rostov-on-Don, Russia \\ c Department of Civil Engineering, University of Rome "Tor Vergata", \\ 00133 Rome, Italy
}

To cite this article: V. Yu. Topolov, S. E. Filippov \& P. Bisegna (2012): Anisotropy Factors and Hydrostatic Parameters of 1-3-Type Piezo-Active Composites with Auxetic Polymer Matrices, Ferroelectrics, 432:1, 92-102

To link to this article: http:// dx. doi.org/ 10.1080/00150193.2012.707890

\section{PLEASE SCROLL DOWN FOR ARTICLE}

Full terms and conditions of use: http://www.tandfonline.com/page/terms-and-conditions

This article may be used for research, teaching, and private study purposes. Any substantial or systematic reproduction, redistribution, reselling, loan, sub-licensing, systematic supply, or distribution in any form to anyone is expressly forbidden.

The publisher does not give any warranty express or implied or make any representation that the contents will be complete or accurate or up to date. The accuracy of any instructions, formulae, and drug doses should be independently verified with primary sources. The publisher shall not be liable for any loss, actions, claims, proceedings, demand, or costs or damages whatsoever or howsoever caused arising directly or indirectly in connection with or arising out of the use of this material. 


\title{
Anisotropy Factors and Hydrostatic Parameters of 1-3-Type Piezo-Active Composites with Auxetic Polymer Matrices
}

\author{
V. YU. TOPOLOV,${ }^{1, *}$ S. E. FILIPPOV,${ }^{2}$ AND P. BISEGNA ${ }^{3}$ \\ ${ }^{1}$ Department of Physics, Southern Federal University, 344090 Rostov-on-Don, \\ Russia \\ ${ }^{2}$ Scientific Design \&Technology Institute "Piezopribor", Southern Federal \\ University, 344090 Rostov-on-Don, Russia \\ ${ }^{3}$ Department of Civil Engineering, University of Rome "Tor Vergata”, 00133 \\ Rome, Italy
}

\begin{abstract}
Results on electromechanical properties and their anisotropy in 1-3-type composites with auxetic polyethylene matrices (Poisson's ratios from -0.83 to-0.20) are reported. The role of elastic properties of the auxetic component in forming a large piezoelectric anisotropy and hydrostatic piezoelectric coefficients $d_{h}^{*} \approx(1000-1500) p C / N$ and $g_{h}^{*} \approx$ $(500-600) \mathrm{mV} \cdot \mathrm{m} / \mathrm{N}$, figures of merit, and electromechanical coupling factors is studied. Condition $d_{33}^{*} / d_{31}^{*} \rightarrow \pm \infty$ is valid at the volume fraction of ceramic $0.22 \leq m^{*} \leq 0.33$, and $m^{*}$ correlates with the ratio $c_{11} \wedge c_{12} \mid$ of elastic moduli of polymer. The effect of the aspect ratio of the elliptic cross section of rods on the volume-fraction dependence of $d_{3 j}^{*}$, the piezoelectric anisotropy, and hydrostatic response is analysed.
\end{abstract}

Keywords 1-3-type composite; electromechanical properties; large piezoelectric anisotropy; auxetic polymer; hydrostatic piezoelectric response

PACS 77.65.-j: Piezoelectricity and electromechanical effects; 77.84.Lf: Composite materials; and 77.84.-s: Dielectric; piezoelectric; ferroelectric; and antiferroelectric materials

\section{Introduction}

Piezo-active composites based on poled ferroelectric ceramics (FCs) have been intensively developed in the last decades and effectively used in various applications. An increase in piezoelectric sensitivity, electromechanical coupling and hydrostatic response of the conventional $\mathrm{FC} /$ polymer composites is often achieved owing to improving the characteristics of the polymer component [1-3]. In the composite with 1-3 connectivity [4], FC rods, distributed continuously along one of the co-ordinate axes, are surrounded by a polymer matrix that is distributed continuously along the three co-ordinate axes. The 1-3-type composites are of value due to their considerable electromechanical coupling and hydrostatic response [1-3,5] and suitable for transducer, hydroacoustic, and other applications. An important opportunity for improving the performance of the 1-3-type composites based

Received March 14, 2012.

*Corresponding author. E-mail: vutopolov@ sfedu.ru 
on FCs consists in use of the so-called auxetic matrix (i.e., the matrix with a negative Poisson's ratio) [6-9] that provides the considerable re-distribution of internal electric and mechanical fields in the composite sample and influences the effective electromechanical properties and related parameters of the studied composite.

In contrast to conventional materials with the positive Poisson's ratios, the auxetic material expands laterally when stretched. Some auxetic polymers and their physical properties are described in a series of experimental papers (see, for instance, Refs. 6, 7, 10, 11). Auxetic behaviour of materials is scale-independent and can be achieved at different structural levels, from molecular to macroscopic levels [11]. As follows from literature data, all isotropic materials (including conventional and auxetic polymers) obey the law of conservation of energy, show a positive definite matrix of elastic compliances (or elastic moduli) and can have the Poisson's ratio in the range from -1 to $0.5[5,8]$. An effect of the auxetic polymer matrix on the performance of the 1-3-type piezo-active composites was discussed in earlier studies $[1,2,7,9]$. For example, according to results [1, 2], the piezo-passive auxetic polymer matrix promotes increasing the hydrostatic piezoelectric coefficient $d_{h}^{*}$ of the 1-3-type composite, and inequalities $d_{h}^{*}>d_{33}^{*}$ and $d_{31}^{*}>0$ hold in certain ranges of the volume fraction of FC. At the same time, no study on the anisotropy of the piezoelectric and other properties of the piezo-active composites was carried out in the context of the auxetic polymer component with variable properties. Changes in elastic properties of polyethylene (PE) - from a conventional high-density modification to a series of microporous auxetic modifications - were shown by Evans and Alderson [7]. Experimental results on auxetic $\mathrm{PE}$ [7] suggest that the radial Poisson's ratio can be varied from -0.20 to -0.83 , and such a large range may be of interest for further studies. In comparison to these data, conventional $\mathrm{PE}$ is characterised by the Poisson's ratio 0.20 [7].

Current literature data on the piezo-active composites show that no comparative study was performed to understand the role of changes in the elastic properties of the auxetic polymer component ${ }^{*}$ ) on the electromechanical properties, their anisotropy and hydrostatic parameters of the FC/polymer composites. The aim of the present paper is to demonstrate interrelations between elastic properties of auxetic $\mathrm{PE}$ and anisotropic electromechanical properties of the 1-3-type FC/PE composites as well to consider the key role of the auxetic polymer component in forming the considerable hydrostatic parameters of these composites.

\section{Model Concepts and Effective Parameters of the 1-3-Type Composite}

Our study is based on the model of the composite that consists of a system of extended cylindrical FC rods aligned parallel to the poling axis $O X_{3}$. These rods are surrounded by a continuous polymer matrix and regularly distributed over a composite sample. In case of a circular cross section of each rod in the $\left(X_{1} O X_{2}\right)$ plane, a square arrangement of the rods in the $\left(X_{1} O X_{2}\right)$ plane is observed. In case of an elliptic cross section, semi-axes $a_{i}$ of each ellipse obey an equation

$$
\left(x_{1} / a_{1}\right)^{2}+\left(x_{2} / a_{2}\right)^{2}=1,
$$

and a rectangular arrangement of the rods in the $\left(X_{1} O X_{2}\right)$ plane takes place.

Hereafter the electromechanical properties of the FC and polymer components are denoted with superscripts (1) and (2), respectively. The effective electromechanical properties

\footnotetext{
*) Since various polymers are characterised by dielectric permittivity being two or three ordersof-magnitude less than dielectric permittivity of FCs [12], one can neglect any changes in dielectric permittivity of the auxetic polymer component in the present study.
} 
Table 1

Room-temperature elastic moduli $c_{a b}{ }^{(1), E}$ (in $10^{10} \mathrm{~Pa}$ ), piezoelectric coefficients $e_{i j}{ }^{(1)}$ (in $\mathrm{C} / \mathrm{m}^{2}$ ) and dielectric constants $\varepsilon_{p p}{ }^{(1), \xi} / \varepsilon_{0)}$ of PCR-7M FC [16]. Note that for poled FCs with $\infty \mathrm{mm}$ symmetry, condition $c_{66}^{(1), E}=\left(c_{11}^{(1), E}-c_{12}^{(1), E}\right) / 2$ is valid [17]

\begin{tabular}{cccccccccc}
\hline$c_{11}^{(1), E}$ & $c_{12}^{(1), E}$ & $c_{13}^{(1), E}$ & $c_{33}^{(1), E}$ & $c_{44}^{(1), E}$ & $e_{31}^{(1)}$ & $e_{33}^{(1)}$ & $e_{15}^{(1)}$ & $\varepsilon_{11}^{(1), \xi} / \varepsilon_{0}$ & $\varepsilon_{33}^{(1), \xi} / \varepsilon_{0}$ \\
\hline 13.3 & 9.2 & 9.1 & 12.5 & 2.28 & -9.5 & 31.1 & 20.0 & 1980 & 1810 \\
\hline
\end{tabular}

of the 1-3 composite are determined in the long-wave approximation as a function of the volume fraction $m$ of FC by means of the effective field method (EFM, for the circular cross section of the rod) [13] and finite element method (FEM, for the elliptic cross section of the rod) [14]. In case of the elliptic cross section, a value of the aspect ratio $\eta=a_{2} / a_{1}$ is varied in a range $0<\eta \leq 1$, so that the limiting case of $\eta=1$ is related to the circular cross section. In the aforementioned methods, elastic moduli $c_{a b}^{(n), E}$ measured at $E=\mathrm{const}$, piezoelectric coefficients $e_{i j}^{(n)}$, and dielectric constants $\varepsilon_{p p}^{(n), \xi}$ measured at mechanical strain $\xi=$ const are used to determine the effective electromechanical properties of the composite, i.e., $c_{a b}^{* E}, e_{i j}^{*}$, and $\varepsilon_{p p}^{* \xi}$. The effective electromechanical properties of the 1-3-type composite are determined within the framework of the EFM [13] from

$$
\begin{aligned}
& \|C *\|=\left\|C^{(2)}\right\|+m\left(\left\|C^{(1)}\right\|-\left\|C^{(2)}\right\|\right)[\|I\| \\
& \left.\quad+(1-m)\|S\|\left\|C^{(2)}\right\|^{-1}\left(\left\|C^{(1)}\right\|-\left\|C^{(2)}\right\|\right)\right]^{-1}
\end{aligned}
$$

where $\left\|C^{(n)}\right\|=\left(\begin{array}{l}\left\|c^{(n), E}\right\|\left\|e^{(n)}\right\|^{t} \\ \left\|e^{(n)}\right\|-\left\|\varepsilon^{(n), \xi}\right\|\end{array}\right)$ is the $9 \times 9$ matrix that characterises the electromechanical properties of the rods $(n=1)$ and the surrounding medium $(n=2)$, superscript $t$ denotes the transposition, $\|I\|$ is the $9 \times 9$ identity matrix, $\|S\|$ is the $9 \times 9$ matrix that contains components of the Eshelby electroelastic tensor [15].

Among components to be of interest for our study, we choose the soft PZT-type PCR-7M composition (PCR is the abbreviation for the group "piezoelectric ceramics from Rostov-on-Don" [16], see constants of PCR-7M FC in Table 1) and a few polyethylene (PE) specimens [7] with variable elastic properties (Table 2). It should be noted that PCR-7M

\section{Table 2}

Room-temperature elastic moduli $c_{a b}^{(2)}$ (in $10^{9} \mathrm{~Pa}$ ) and Poisson's ratios $v^{(2)}$ of auxetic microporous and monolithic specimens of PE [7]. Notations of auxetic components are taken from work [7]

\begin{tabular}{lccc}
\hline Polymer component & $c_{11}^{(2)}$ & $c_{12}^{(2)}$ & $v^{(2)}$ \\
\hline Auxetic PE-1 & 1.82 & -0.801 & -0.20 \\
Auxetic PE-4 & 0.342 & -0.0871 & -0.35 \\
Auxetic PE-6 & 0.278 & -0.0861 & -0.45 \\
Auxetic PE-8 & 0.505 & -0.185 & -0.58 \\
Auxetic PE-9 & 0.769 & -0.349 & -0.83 \\
Conventional & & & \\
$\quad$ high-density PE & 0.778 & 0.195 & 0.20 \\
\hline
\end{tabular}


FC is characterised [16] by high piezoelectric activity (piezoelectric coefficients $d_{33}^{(1)}=755$ $\mathrm{pC} / \mathrm{N}$ and $\left.d_{31}^{(1)}=-347 \mathrm{pC} / \mathrm{N}\right)$ and a moderate anisotropy of $d_{3 j}^{(1)}\left(d_{33}^{(1)} / d_{31}^{(1)}=-2.18\right)$. For the PE specimens listed in Table 2, we assume that their dielectric permittivity $\varepsilon_{p p}^{(2)} / \varepsilon_{0}=2.3$ (as is known for monolithic PE [12]) and $\varepsilon_{p p}^{(2)} \ll \varepsilon_{p p}^{(1)}$ at $p=1$ and 3. Taking into account the microporous microgeometry of auxetic PE specimens [7], we characterise the studied composite by 1-3-0 connectivity.

Based on calculated values of $c_{a b}^{* E}, e_{i j}^{*}$ and $\varepsilon_{p p}^{* \xi}$ of the composite and using conventional formulae [17] for the piezoelectric medium, we evaluate a series of effective parameters of the composite. These parameters are divided into three groups. The first group represents the longitudinal parameters, such as piezoelectric coefficients $d_{33}^{*}$ and $g_{33}^{*}=d_{33}^{*} / \varepsilon_{33}^{* \sigma}$, electromechanical coupling factor $k_{33}^{*}=d_{33}^{*} /\left(\varepsilon_{33}^{* \sigma} s_{33}^{* E}\right)^{1 / 2}$, squared figure of merit $\left(Q_{33}^{*}\right)^{2}=d_{33}^{*} g_{33}^{*}$, etc. The second group of the parameters characterises the hydrostatic (hydrophone) piezoelectric response of the composite and comprises hydrostatic piezoelectric coefficients $d_{h}^{*}=d_{33}^{*}+d_{32}^{*}+d_{31}^{*}$ and $g_{h}^{*}=d_{h}^{*} / \varepsilon_{33}^{* \sigma}$, hydrostatic electromechanical coupling factor $k_{h}^{*}=$ $d_{h}^{*} /\left(\varepsilon_{33}^{* \sigma} s_{h}^{* E}\right)^{1 / 2}$, squared hydrostatic figure of merit $\left(Q_{h}^{*}\right)^{2}=d_{h}^{*} g_{h}^{*}$, etc., where $s_{h}^{* E}=$ $\sum_{a, b=1}^{3} s_{a b}^{* E}$ is hydrostatic elastic compliance at $E=$ const, and electrodes on the composite sample are parallel to the $\left(X_{1} O X_{2}\right)$ plane. The third group of the parameters is represented by two anisotropy factors, $\zeta_{d}=d_{33}^{*} / d_{31}^{*}$ and $\zeta_{k}=k_{33}^{*} / k_{31}^{*}$. For the composite with the elliptic cross section of the FC rods, additional ratios $d_{33}^{*} / d_{32}^{*}$ and $k_{33}^{*} / k_{32}^{*}$ can be introduced following concepts of paper [14]. It should be mentioned that the piezoelectric coefficients $d_{3 j}^{*}$ and $d_{h}^{*}$ are used to describe piezoelectric activity of the composite, the piezoelectric coefficients $g_{3 j}^{*}$ and $g_{h}^{*}$ characterise piezoelectric sensitivity. Squared figures of merit $\left(Q_{33}^{*}\right)^{2}$ and $\left(Q_{h}^{*}\right)^{2}$ are related to power densities and signal-to-noise ratios in piezoelectric transducers, hydrophones and other piezotechnical devices. Electromechanical coupling factors $k_{3 j}^{*}$ and $k_{h}^{*}$ characterise an effectiveness of the conversion of mechanical energy into electric one and vice versa.

Below we consider examples of an original behaviour of the effective parameters from the aforementioned groups and discuss some reasons for this behaviour.

\section{Results and Discussion}

\subsection{Effective Parameters of Composites Versus Elastic Properties of Auxetic Components}

Among the effective parameters from the first and second groups, of particular interest are the parameters that are characterised by non-monotonic volume-fraction dependences. Data on maxima of these parameters (Table 3 ) suggest that only values of max $d_{h}^{*}$ are achieved at volume fractions $m>0.03$. We mention for comparison that the $1-3 \mathrm{FC} /$ polymer composites with volume fractions $m \approx 0.033$ and more have been manufactured and studied earlier [18]. Relatively small values of $m$ (see Table 3 ) are accounted for by the strong influence of the large differences between elastic moduli of the FE and polymer components (irrespective of $v^{(2)}$ ) and by the important role of dielectric permittivity $\varepsilon_{33}^{* \sigma}$ in forming the volumefraction dependence of $g_{33}^{*}, g_{h}^{*},\left(Q_{33}^{*}\right)^{2},\left(Q_{h}^{*}\right)^{2}$, and $k_{h}^{*}$ at $m<0.1$. Thus, for piezotechnical applications it is reasonable to know values of these parameters at $m>0.03$ and trends in decreasing them with increasing $m$. Data from Table 4 show these trends for the studied 


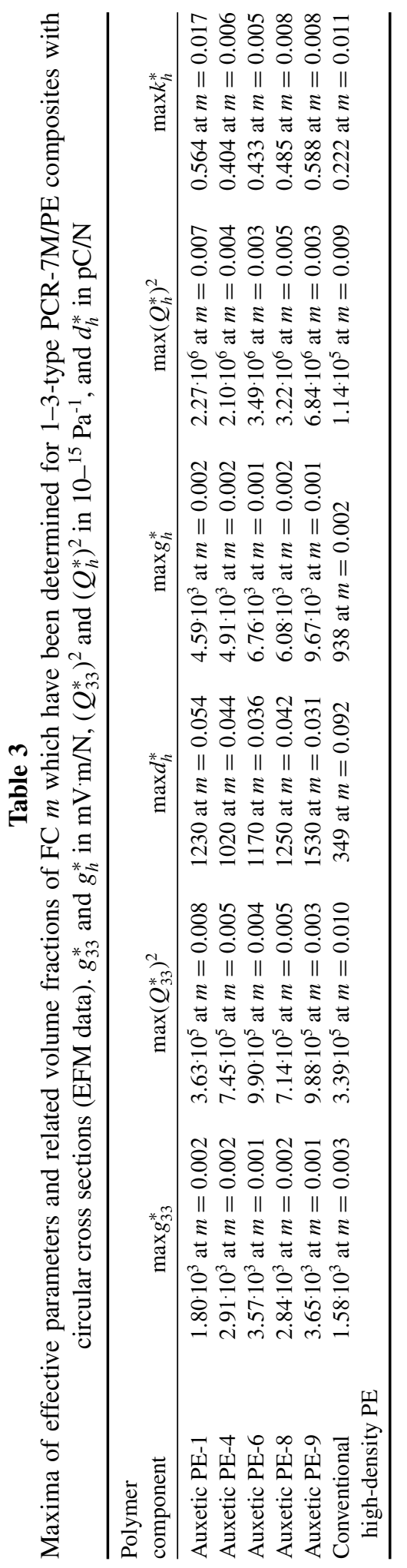




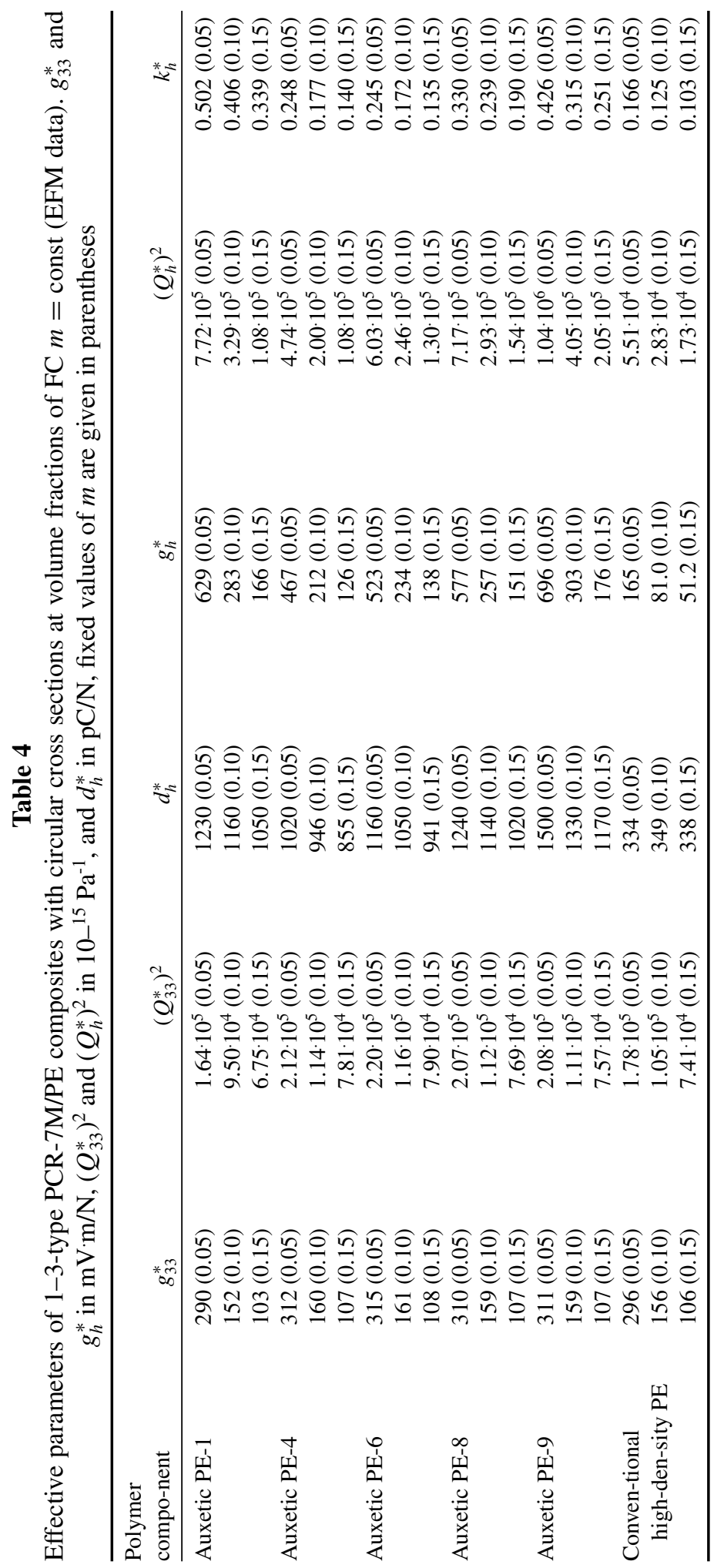


composite with the circular cross section of rods. It is clear that the volume-fraction range $0.03<m<0.10$ is of value for various applications.

Comparison of data from Tables 4 and 2 enables us to emphasise the following features:

(i) largest values of $k_{h}^{*}$ at $m \leq 0.15$ are achieved in the composite with auxetic PE-1 (i.e., polymer with the largest value of $\mathrm{I}_{12}^{(2)} \mid$ from Table 2),

(ii) largest values of $g_{33}^{*}$ and $\left(Q_{33}^{*}\right)^{2}$ are concerned with auxetic PE-6 (i.e., polymer with the smallest value of ${ }_{11}^{(2)}$ from Table 2), and

(iii) largest values of $d_{h}^{*}, g_{h}^{*}$ and $\left(Q_{h}^{*}\right)^{2}$ take place in the presence of auxetic PE-9 (i.e., polymer with the largest value of $\left|v^{(2)}\right|$ from Table 2).

In items (i)-(iii), the elastic constant of the auxetic component plays the important role in achieving the large effective parameters of the studied composite. We mention for comparison that the largest values of hydrostatic parameters predicted for a 1-3-0 PZT FC/auxetic polyurethane composite are $d_{h}^{*}=1458 \mathrm{pC} / \mathrm{N},\left(Q_{h}^{*}\right)^{2}=5.655 \cdot 10-^{9} \mathrm{~Pa}^{-1}$, and $k_{h}^{*}=0.567[1]$.

\subsection{Large Piezoelectric Anisotropy of Composites}

An important feature of the studied composites with auxetic polymer components is the non-monotonic volume-fraction dependence of anisotropy factors $\zeta_{d}=d_{33}^{*} / d_{31}^{*}$ and

$$
\zeta_{\mathrm{k}}=k_{33}^{*} / k_{31}^{*}=\zeta_{\mathrm{d}}\left(s_{11}^{* E} / s_{33}^{* E}\right)^{1 / 2}
$$

Values of $\zeta_{d} \rightarrow \pm \infty$ and $\zeta_{k} \rightarrow \pm \infty$ (Fig. 1a, ) are achieved at a critical volume fraction $m^{*}$ that obeys condition $d_{31}^{*}\left(m^{*}\right)=0$. We show for comparison Fig. 1b, built for the anisotropy factors $\zeta_{d}$ and $\zeta_{k}$ of the related composite with the monolithic PE matrix: in this case $d_{31}^{*}$ $<0$ at any volume fractions $m$, and only $\zeta_{k}$ is characterised by a non-monotonic behaviour. Thus, changes in the Poisson ratio of the polymer matrix $\left(v^{(2)}=-0.20\right.$ in data from Fig. 1a, and $v^{(2)}=0.20$ in data from Fig. $1 \mathrm{~b}$, ) leads to drastic changes in the anisotropy factors of the composite. At the same time, volume-fraction dependences $d_{33}^{*}(m)$ and $k_{33}^{*}(m)$ (Fig. 2)
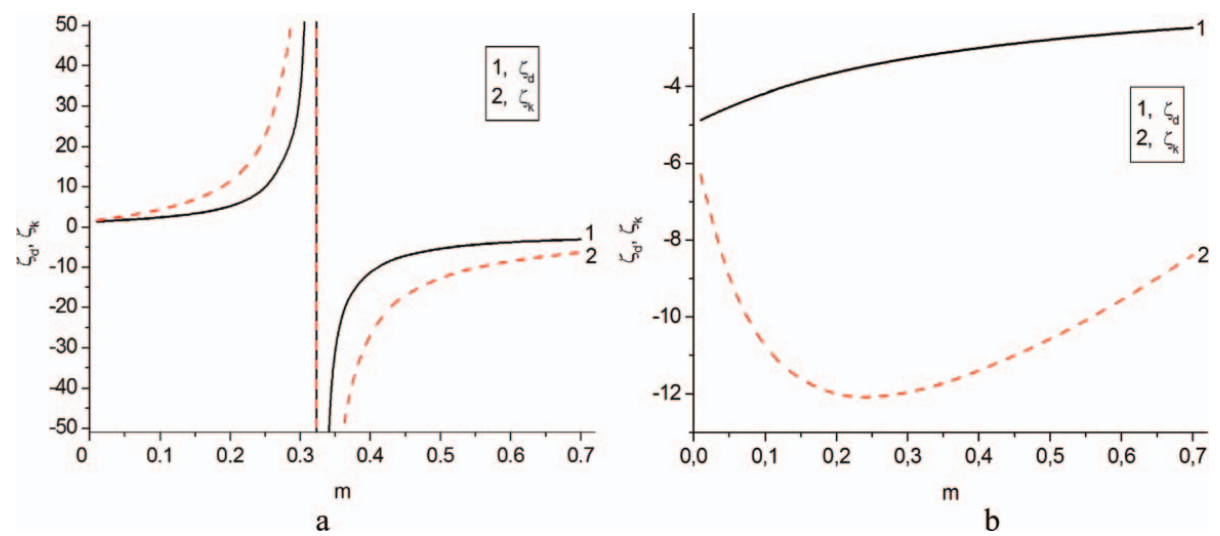

Figure 1. Volume-fraction dependences of anisotropy factors $\zeta_{d}$ and $\zeta_{k}$ which have been calculated by means of the EFM for the following 1-3-type composites with circular cross sections of rods: PCR7M/auxetic PE-1 (a, 1-3-0 connectivity) and PCR-7M/PE (b, 1-3 connectivity) (Figure available in color online). 


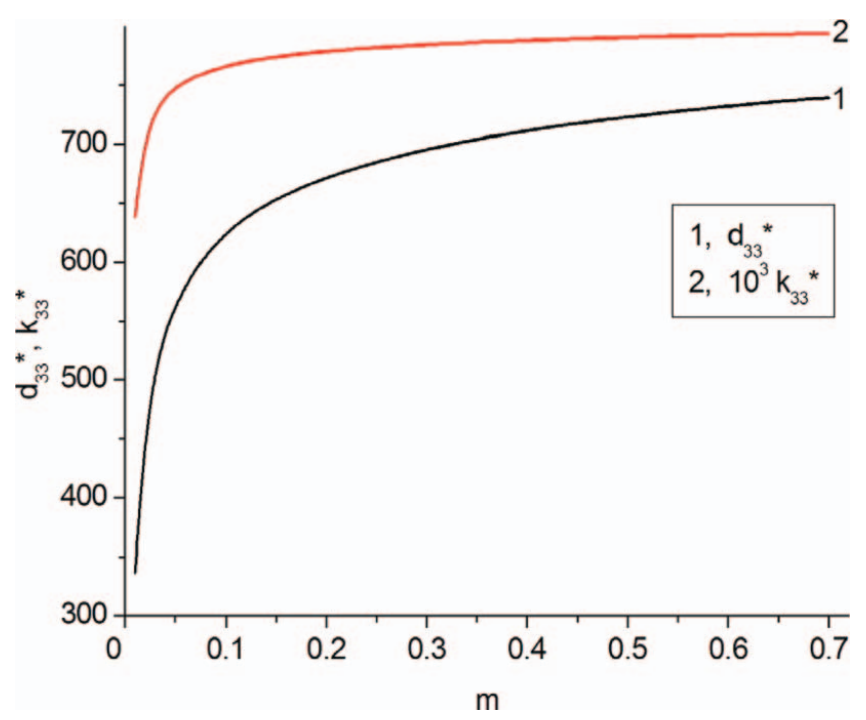

Figure 2. Volume-fraction dependences of piezoelectric modulus $d_{33}^{*}($ in $\mathrm{pC} / \mathrm{N}$ ) and electromechanical coupling factor $k_{33}^{*}$ which have been calculated by means of the EFM for the 1-3-0 PCR$7 \mathrm{M} /$ auxetic PE-1 composite with circular cross sections of rods (Figure available in color online).

undergo relatively small changes (less than 5\%) at replacing the PE matrix and changing its $v^{(2)}$ value, even if to choose the conventional PE component with $v^{(2)}>0$ instead of the auxetic component with $v^{(2)}<0$. The same small changes in $d_{33}^{*}(m)$ and $k_{33}^{*}(m)$ are also observed if to replace the auxetic PE matrix with the monolithic PE matrix. The ratio

\section{Table 5}

Anisotropy factors $\zeta_{d}$ and $\zeta_{k}$ and critical volume fractions $m^{*}$, which are related to 1-3-type PCR-7M/auxetic PE composites with circular cross sections (EFM data, fixed values of $m$ are given in parentheses), and ratios of elastic moduli $c_{11}^{(2)} /\left|c_{12}^{(2)}\right|$ of auxetic PE

\begin{tabular}{lcccc}
\hline Polymer component & $\zeta_{d}$ & $\zeta_{k}$ & $m^{*}$ & $c_{11}^{(2)} /\left|c_{12}^{(2)}\right|$ \\
\hline Auxetic PE-1 & $1.71(0.05)$ & $2.63(0.05)$ & 0.324 & \\
& $2.32(0.10)$ & $4.30(0.10)$ & & 2.28 \\
Auxetic PE-4 & $3.30(0.15)$ & $6.77(0.15)$ & & \\
& $4.02(0.05)$ & $11.5(0.05)$ & 0.220 & \\
Auxetic PE-6 & $6.15(0.10)$ & $22.9(0.10)$ & & 3.86 \\
& $11.3(0.15)$ & $48.6(0.15)$ & & \\
Auxetic PE-8 & $3.04(0.05)$ & $9.62(0.05)$ & 0.252 & \\
& $4.39(0.10)$ & $18.2(0.10)$ & & \\
& $7.06(0.15)$ & $33.7(0.15)$ & & \\
Auxetic PE-9 & $2.32(0.05)$ & $5.72(0.05)$ & 0.285 & \\
& $3.24(0.10)$ & $10.2(0.10)$ & & \\
& $4.83(0.15)$ & $17.4(0.15)$ & & \\
& $1.61(0.05)$ & $3.42(0.05)$ & 0.330 & \\
& $2.19(0.10)$ & $5.85(0.10)$ & & \\
& $3.09(0.15)$ & $9.33(0.15)$ & & \\
\hline
\end{tabular}


of elastic moduli of components $c_{33}^{(1), E} / c_{33}^{(2)}=c_{33}^{(1), E} /_{11}^{(2)} \gg 1$ plays the important role in forming the longitudinal piezoelectric effect in the 1-3-type composite and, therefore, the volume-fraction dependences $d_{33}^{*}(m)$ and $k_{33}^{*}(m)$. Small changes in the ratio of $c_{33}^{(1), E} / c_{33}^{(2)}$ at replacing the PE matrix (cf. data from Tables 1 and 2) would not considerably affect curves of $d_{33}^{*}(m)$ and $k_{33}^{*}(m)$ from Fig. 2.

Data on the anisotropy factors $\zeta_{d}$ and $\zeta_{k}$ of composites with auxetic PE matrices at $0.05 \leq m<m^{*}$ (Table 5) suggest that

(i) $\zeta_{k}>\zeta_{d}$ (this inequality is accounted for by the ratio $s_{11}^{* E} / s_{33}^{* E}>1$ in Eq. (2), and such behaviour of elastic compliances $s_{a a}^{* E}$ of the composite is caused by the presence of the $\mathrm{FC}$ rods oriented along the $\mathrm{OX}_{3}$ axis) and

(ii) decreasing the critical volume fraction $m^{*}$ is observed at increasing the ratio $c_{11}^{(2)} /$ $\left|c_{12}^{(2)}\right|$ related to the auxetic component.

As a consequence, auxetic PE-4 with the largest value of $c_{11}^{(2)} /\left|c_{12}^{(2)}\right|$ provides the largest anisotropy factors $\left(\zeta_{d}>10\right.$ and $\left.\zeta_{k}>10\right)$ at $m \approx 0.15$. It should be added that the composite at this volume fraction of ceramic is characterised by relatively large values of $d_{33}^{*}(m)$ and

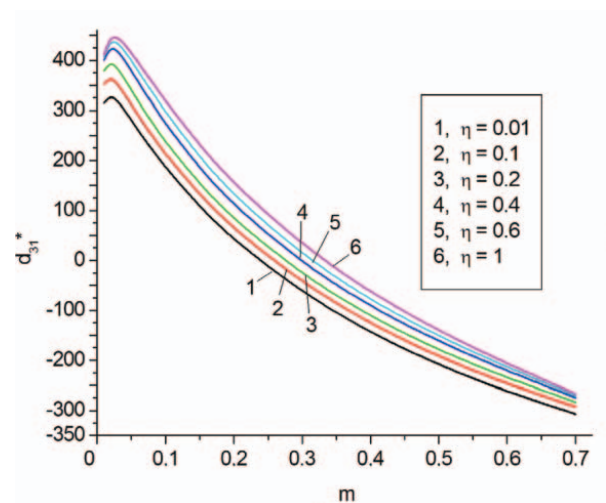

a

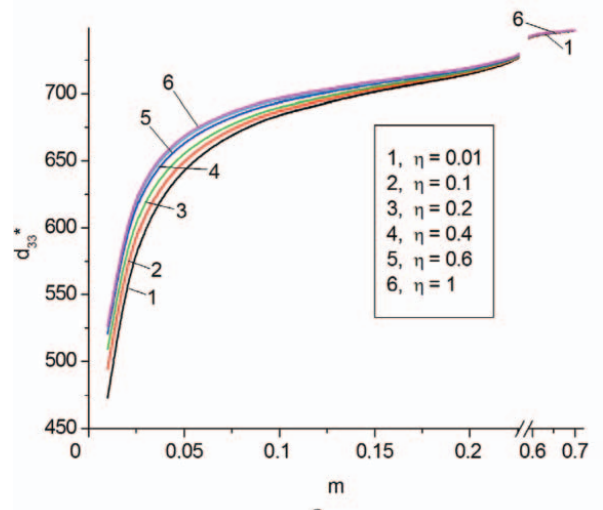

c

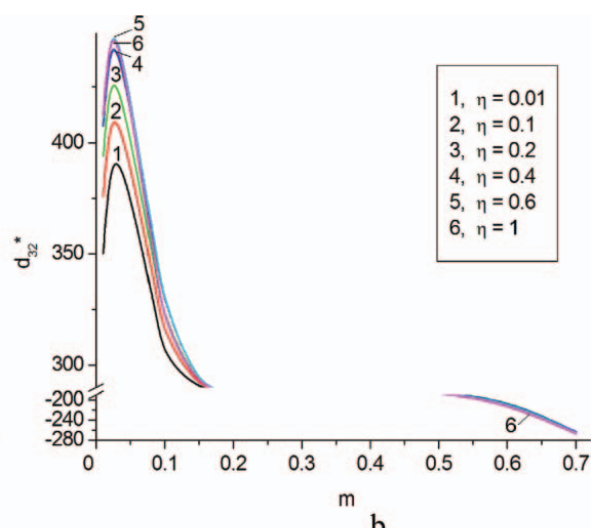

b

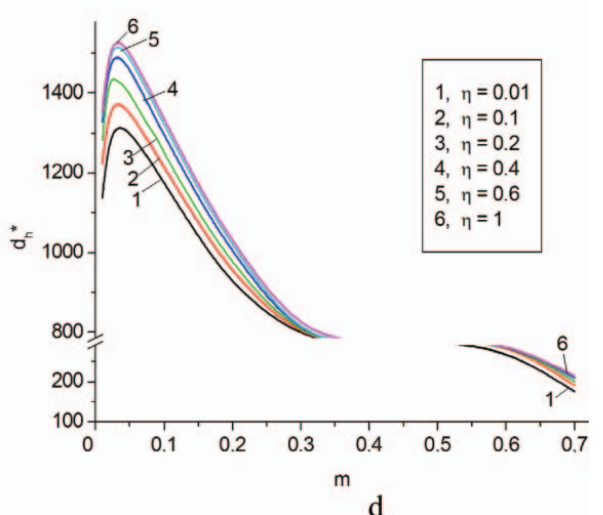

d

Figure 3. Volume-fraction dependences of piezoelectric moduli $d_{3 j}^{*}(\mathrm{a}-\mathrm{c}$, in $\mathrm{pC} / \mathrm{N})$ and $d_{h}^{*}(\mathrm{~d}$, in $\mathrm{pC} / \mathrm{N}$ ) which have been calculated by means of the FEM for the 1-3-0 PCR-7M/auxetic PE-9 composite with elliptic cross sections of rods. 
$k_{33}^{*}(m)$ (see, e.g., Fig. 2 for the composite with auxetic PE-1) and, therefore, has obvious advantages over high-anisotropic $\mathrm{FCs}$ of the $\mathrm{PbTiO}_{3}$ type [19]. The studied composite based on the PZT-type FC has no analogues among the known piezo-active materials and can be used as a material with large values of $d_{33}^{*}, k_{33}^{*}, \zeta_{d}$, and $\zeta_{k}$ which are obtained in relatively wide ranges of $m$.

The change in the aspect ratio $\eta$ of the elliptic cross section of the FC rod influences both the piezoelectric anisotropy and hydrostatic response of the composite (Fig. 3). Graphs in Fig. $3 \mathrm{a}$ and $\mathrm{b}$, suggest that the largest maximum values of $d_{3 j}^{*}(m)>0(j=1$ and 2$)$ are achieved at $\eta=1$, i.e., in the presence of the circular cylindrical FC rods, and such microgeometry of the composite promotes the larger hydrostatic piezoelectric coefficient $d_{h}^{*}(m)$ (curve 6 in Fig. 3d, ). Changes in the aspect ratio $\eta$ give rise to inessential changes in $d_{33}^{*}(m)$ (Fig. 3c, ) due to the weak effect of the cross section of the rod on the lateral piezoelectric activity of the 1-3-type composite. Data from Fig. 3c, d, suggest that values of $d_{h}^{*} \approx(1000 \ldots 1500) \mathrm{pC} / \mathrm{N}$ and $d_{h}^{*} \approx 2 d_{33}^{*}$ are achieved in the relatively wide volumefraction range and can be used in hydroacoustic, sensor and actuator applications. This circumstance and data from Table 4 can be regarded as main advantages of the studied composite with the auxetic component.

Our comparison of the data calculated for the 1-3-type composites at $\eta=1$ shows that the electromechanical properties $\left(c_{a b}^{* E}, e_{i j}^{*}\right.$, and $\left.\varepsilon_{p p}^{* \xi}\right)$ determined using the EFM well agree with the similar FEM evaluations at $0<m \leq 0.7$. As follows from numerous evaluations, differences between electromechanical constants calculated using the aforementioned methods are less than $5 \%$.

\section{Conclusions}

The comparative study of the electromechanical properties, anisotropy factors and hydrostatic parameters of the 1-3-type FC/auxetic polymer composites has been carried out taking into account changes in elastic properties of the auxetic component. The effective electromechanical properties and related parameters of these composites have been predicted in the wide volume-fraction range by means of the EFM and FEM. The auxetic PE matrix with the negative Poisson's ratio promotes relatively large values of maxima of effective parameters of the composites (e.g., $g_{33}^{*},\left(Q_{33}^{*}\right)^{2}, g_{h}^{*},\left(Q_{h}^{*}\right)^{2}, d_{h}^{*}$, and $\left.k_{h}^{*}\right)$, and volume-fraction dependences of these parameters enable one to use them in wide volume fractions. It has been shown how to select the proper auxetic component for obtaining the larger effective parameter of the composite, for instance, $\max d_{h}^{*}=1530 \mathrm{pC} / \mathrm{N}$. While the FC component shows the piezoelectric anisotropy $d_{33}^{(1)} / d_{31}^{(1)}=-2.18$, the auxetic component promotes $d_{31}^{*}=0$ and $d_{32}^{*}=0$ in the studied composites, and it opens up new possibilities of use of the high-anisotropic and high-active piezoelectric materials (with $\zeta_{d} \gg 1$ and $\zeta_{k} \gg 1$ at $d_{33}^{*} \approx 700 \mathrm{pC} / \mathrm{N}$ and $k_{33}^{*} \approx 0.75$ ). Moreover, the PZT-type FC component with high piezoelectric activity provides the large values of $d_{33}^{*}$ and $k_{33}^{*}$ even at relatively small volume fractions of FC $(m \approx 0.1)$. The results of the present study can be of interest for those manufacturing the novel anisotropic piezo-active composites and predicting the effective parameters of these composites for various piezotechnical applications.

\section{Acknowledgments}

The authors would like to thank Prof. Dr. C. R. Bowen (University of Bath, UK), Prof. Dr. A. E. Panich and Prof. Dr. I. A. Parinov (Southern Federal University, Rostov-on-Don, Russia), and Prof. Dr. M. Lethiecq and Dr. F. Levassort (François Rabelais University, 
Tours, France) for their continuing interest in the research problems. This work has been carried out with the financial support from the Ministry of Education and Science of Russia within the framework of the Federal Purposive Programme entitled "Studies and Working out on Priority Directions of the Development of the Research Complex of Russia" for 2007-2013.

\section{References}

1. L. V, Gibiansky, and S. Torquato, On the use of the homogenization theory to design optimal piezocomposites for hydrophone applications. J. Mech. Phys. Solids. 45, 689-708 (1997).

2. V. Yu. Topolov, and C. R. Bowen, Characteristics of 1-3-type ferroelectric ceramic/auxetic polymer composites. Modelling Simul. Mater. Sci. Eng. 16, 015007-12 (2008).

3. V. Yu. Topolov, S. E. Filippov, and A. A. Vorontsov, Piezoelectric effect and anisotropy of electromechanical properties of novel 1-0-3 composites based on ferroelectric ceramics. Nanoi Mikrosistemnaya Tekhnika Nr. 9: 13-19 (in Russian) (2011).

4. R. E. Newnham, D. P. Skinner, and L. E. Cross, Connectivity and piezoelectric-pyroelectric composites. Mater. Res. Bull. 13, 525-536 (1978).

5. A. Safari, and E. K. Akdogan, Rapid prototyping of novel piezoelectric composites. Ferroelectrics 331, 153-179 (2006).

6. J. B. Choi, and R. Lakes, Nonlinear properties of polymer cellular materials with a negative Poisson's ratio. J. Mater. Sci. 27, 4678-4684 (1992).

7. K. E. Evans, and K. L. Alderson, The static and dynamic moduli of auxetic microporous polyethylene. J. Mater. Sci. Lett. 11, 1721-1724 (1992).

8. W. A. Smith, Optimizing electromechanical coupling in piezocomposites using polymers with negative Poisson's ratio. In: Proc. IEEE Ultrasonics Symp. Dec. 8-11, 1991, Lake Buena Vista, FL, USA. V. 1. New York: IEEE; 661-666 (1991).

9. Y. C. Wang, and R. S. Lakes, Extreme thermal expansion, piezoelectricity, and other coupled field properties in composites with a negative stiffness phase. J. Appl. Phys. 90, 6458-6465 (2001).

10. W. Yang, Z-M. Li, W. Shi, and B-H. Xie, M-B. Yang, Review on auxetic materials. J. Mater. Sci. 39, 3269-3279 (2004).

11. Y. Liu, and H. Hu, A review on auxetic structures and polymeric materials. Sci. Res. Essays 5, 1052-1063 (2010).

12. I. N. Groznov, Dielectric permittivity. In: Physics Encyclopaedia. Moscow: Sovetskaya Entsiklopediya; 178-179 (in Russian) (1983).

13. V. M. Levin, M. I. Rakovskaja, and WS. Kreher, The effective thermoelectroelastic properties of microinhomogeneous materials. Internat. J. Solids. Struct. 36, 2683-2705 (1999).

14. V. Yu. Topolov, and P. Bisegna, Anisotropic piezoelectric properties of 1-3 ceramic/polymer composites comprising rods with elliptic cross section. J. Electrocer. 25, 26-37 (2010).

15. J. H. Huang, and S. Yu, Electroelastic Eshelby tensors for an ellipsoidal piezoelectric inclusion. Composites Engin. 4: 1169-1182 (1994).

16. A. Y. a. Dantsiger, O. N. Razumovskaya, L. A. Reznitchenko, L. D. Grineva, R. U. Devlikanova, S. I. Dudkina, S. V. Gavrilyatchenko, N. V. Dergunova, and A. N. Klevtsov, Highly Effective Piezoceramic Materials (Handbook). Rostov-on-Don: Kniga; (in Russian) (1994).

17. T. Ikeda, Fundamentals of Piezoelectricity. Oxford, New York, Toronto: Oxford University Press; (1990).

18. S. H. Choy, H. L. W. Chan, M. W. Ng, and P. C. K. Liu, Study of 1-3 PZT fibre/epoxy composites with low volume fraction of ceramics. Integr. Ferroelectrics 63, 109-115 (2004).

19. S. Ikegami, I. Ueda, and T. Nagata, Electromechanical properties of $\mathrm{PbTiO}_{3}$ ceramics containing La and Mn. J. Acoust. Soc. Am. 50, 1060-1066 (1971). 